Perspective

\title{
Femtosecond assisted lasik for post PKP myopic astigmatism
}

\section{Introduction}

High residual refractive error can be a disappointing end point to an otherwise successful penetrating keratoplasty, it can prevent the patient attaining satisfactory visual acuity.

\section{Causes of astigmatism post PKP}

i. Size and shape difference between donor and host

ii. Eccentric placement

iii. Scarring at graft host junction

iv. Asymmetric forces in the cornea resulting in altered shape
Astigmatic patterns
i. According to ESCRS istanbul (Dr Rudy niujts)
ii. 4 to $5 \mathrm{D}$ and anisometropia
iii. $30 \%$ shows $>5 \mathrm{D}$
iv. $25 \%$ regular
v. $75 \%$ irregular

\section{Vision correction options after keratoplasty}

Non surgical

i. Spectacles.

ii. Contact lenses

iii. Surgical

iv. PRK ... (disadvantage) haze and scar.

v. Corneal relaxing incision, wedge resection, along with \pm compression sutures........ (disadvantage) Wound dehiscence, corneal topographic changes, low predictability of the results, lack of internationally accepted nomograms.

vi. Lasik using microkeratome or femtosecond laser.

vii. Implantable contact lenses.

viii. ICRS

ix. Femtosecond laser-assisted

$\mathrm{x}$. Intrastromal corneal ring

xi. Segment implantation for high

xii. Astigmatism correction after

xiii. Penetrating keratoplasty

xiv. Carlos Lisa MD, Miriam García-Fernández MD, David MadridCosta $\mathrm{PhD}$, Leonardo Torquetti MD, PhD, Jesús Merayo-Lloves $\mathrm{MD}, \mathrm{PhD}$, José Fernando Alfonso MD, PhD correspondence mail

\author{
Volume 6 Issue 2 - 2017
}

\section{Bahaa Al Din Jaber}

Department of Cornea Refractive Surgery, Bahaa Eye Clinic, Jordon

Correspondence: Bahaa AI Din Jaber, Department of Cornea Refractive Surgery, Bahaa Eye Clinic Amman Jordan, Email DOCTORB_22@hotmail.com

Received: January 23, 2017 | Published: February 8, 2017

\section{Femtosecond laser}

Ultra-short pulse duration $(10-15 \mathrm{sec}$.) has the ability to deliver laser energy with minimal collateral damage to the adjacent tissue $(1 \mu \mathrm{m})$.

\section{Femtosecond laser}

i. Mechanism of action

ii. Photo disruption

iii. FSL energy is absorbed by the tissue, resulting in plasma formation.

iv. This plasma of free electrons and ionized molecules rapidly expands, creating cavitation bubbles.

v. The force of the cavitation bubble creation separates the tissue (Figure 1).

\section{Femto laser}

Why femto?

The only reliable and predictable way of creating the flap within the graft.

\section{Why femto (cont'd)}

i. Reduced incidence of flap complications like buttonholes, free caps, irregular cuts etc.

ii. Control over flap diameter and thickness; side cut angle, hinge position and length.

iii. Increased precision with improved flap safety and better thickness predictability.

iv. Capability of cutting thinner flaps to accommodate thin corneas and high refractive errors.

\section{Why femto (cont'd)}
i. Stronger flap adherence.
ii. Better contrast sensitivity.
iii. Decreased incidence of epithelial in growth. 
iv. Extremely low incidence of graft wound dehiscence secondary to less increase in IOP required. (20 to $35 \mathrm{~mm}$ ) compared to microkeratome (55 to 70 ).

v. NB. The fibrotic edge of the graft was also an additional safety factor for us for the prevention of a possible transplant wound rupture.

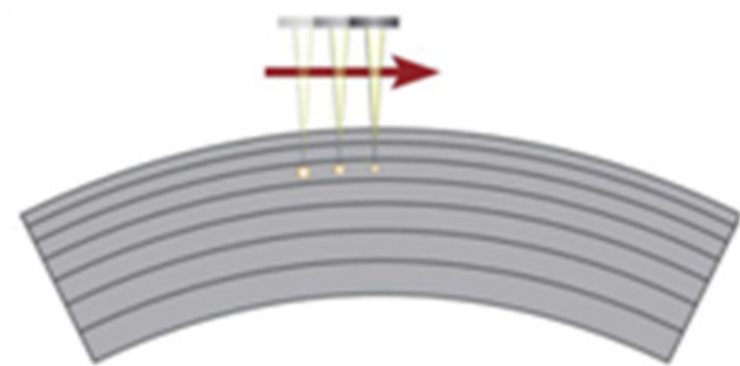

A

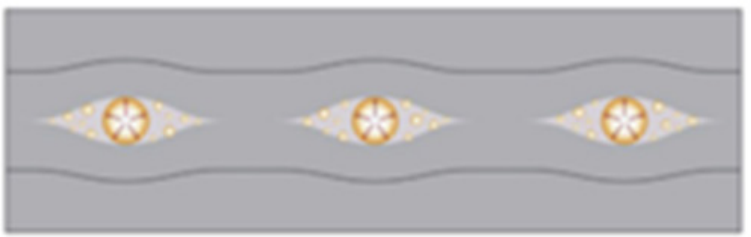

$\boldsymbol{B}$

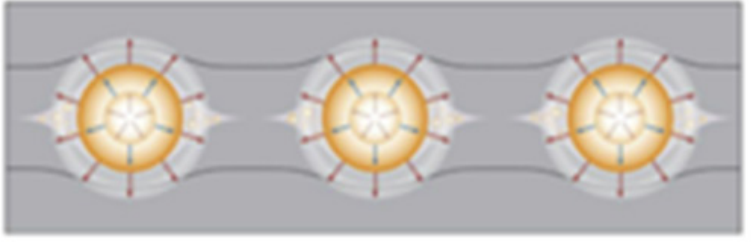

C

Figure I Femto laser.

Why femto (cont'd)

i. Lesser incidence of dry eye due to higher epithelial stability.

ii. Lesser hemorrhage from limbal vessels.

iii. The ability to retreat immediately if there is incomplete FS laser ablation.

\section{Why Femto?}

i. It is capable, to a certain extent, of passing through optically hazy media, such as an edematous cornea.

ii. The laser may be applied in multiple geometric patterns including vertical, spiral, or zigzag cuts.

iii. It can be focused anywhere within or behind the cornea.

\section{Why Femto?}

It is not a blade! (Figure 2).

\section{Aim of the study}

The purpose of this study is to determine the efficacy and stability of LASIK for the correction of post keratoplasty myopic astigmatism using a femtosecond laser for flap creation.

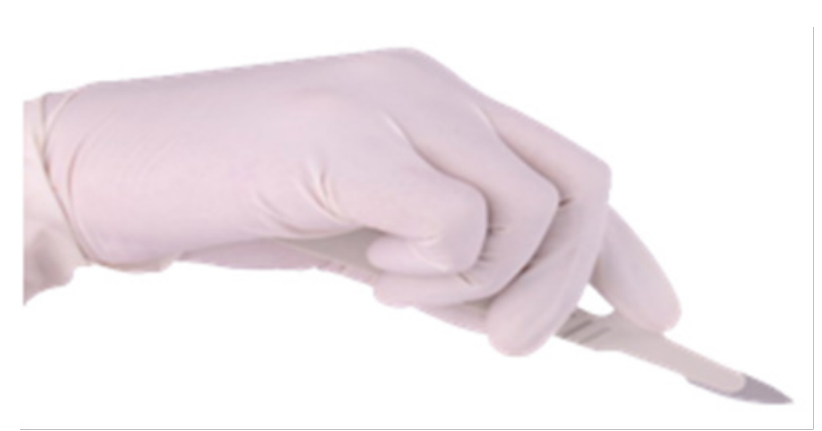

Figure 2 It is not a blade!

\section{Study design}

i. Retrospective study, 40eyes of 35 patients

ii. Time between PKP and femtosecond lasik 18 to 65 months mean (41.5)

iii. Interval at least 8 months after suture removal

iv. AGE: 25years TO 54years MEAN 39.5

v. Follow up 1, 3 and 12months

\section{Study design (Cont'd)}

All patients were examined pre \& post operatively for:

i. UCVA

ii. BSCVA

iii. Autoreffraction and cycloreffaction(cyclo pre only),

iv. Corneal topography(TMS4),

v. Corneal tomographhy (allegretto occulizer),

vi. IOP,

vii. ORA,

viii. And Pachymetry.

\section{Study design (cont'd)}

i. Contraindications for refractive intervention include peripheral corneal vascularization, thin host tissue, wound ectasia and significant graft override or malapposition.

ii. The patient should have a minimal corneal thickness of at least $500 \mu \mathrm{m}$.

iii. The possibility of graft rejection was considered and addressed by the use of topical steroids post operatively.

\section{Pre op}

i. Pre op myopia -3.50 to $-7.75 \mathrm{D}$

ii. Pre op astigmatism -3.00 to $-6.00 \mathrm{D}$

iii. Pre op UCVA CF 3 mtr to 0.30

iv. "The attempted spherical equivalent correction ranged between 60 to $90 \%$ of the measured pre op refraction."

\section{Post op}


i. Myopia -0.75 to $-1.75 \mathrm{D}$

ii. Astigmatism -1.25 to -2.25

iii. UCVA 0.40 TO 0.90

\section{Discussion}

i. In 3 month follow up 75 to $92 \%$ reached the attempted correction $( \pm 1.00 \mathrm{D})$.

ii. (Mean increase is 4lines) 3months.

iii. And in 1 year follow up all of the examined eyes ( 31 out of 40 ) gained at least 3 lines on snellin acuity tests.

iv. No pt has lost one line of his original visual acuity.

v. One eye exhibited peripheral flap epithelial in growth which didn't require any flap re lifting.

\section{Data analysis}

Se pre op vs post op (Figures 3-6).

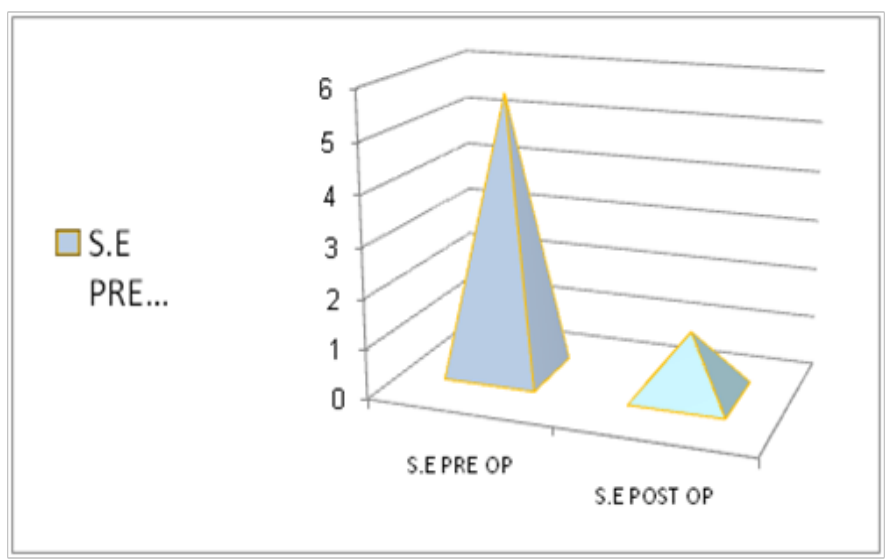

Figure 3 Data Analysis: Se pre op vs post op.

ASTIG

PRE

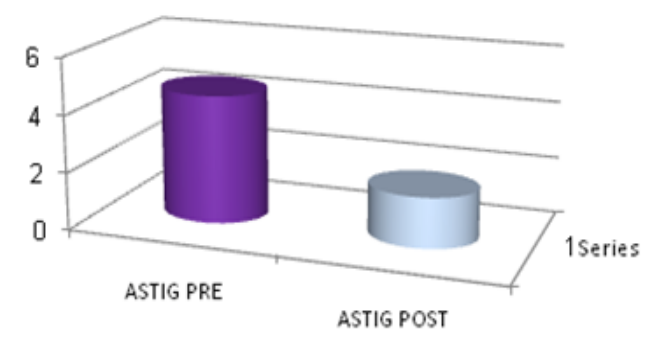

Figure 4 Data Analysis: astig pre vs astig post.

\section{Conclusion}

i. Femto lasik is an effective procedure for pt with myopic astigmatism post PKP.

ii. It is also relatively a safe procedure with minimal complications regarding the surgery itself and the graft. iii. However; more work should be done regarding the endothelial cell count and topographic changes over years.

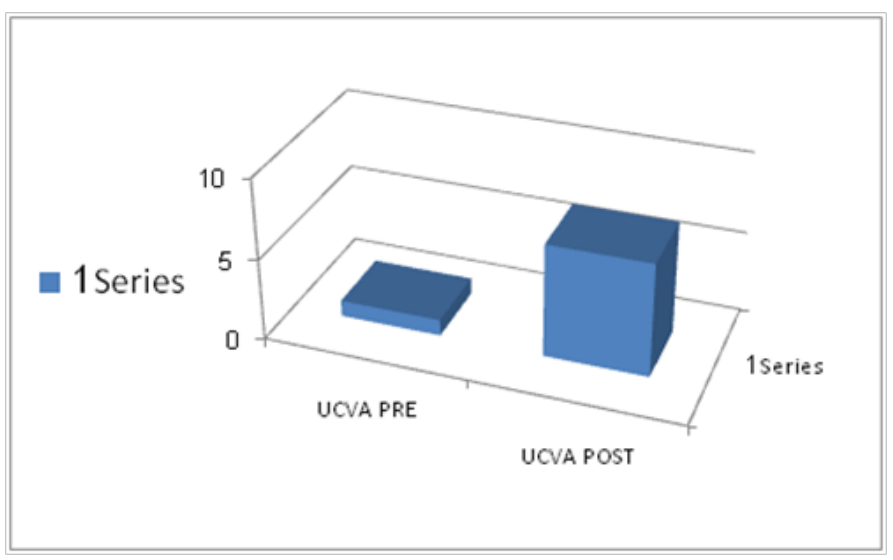

Figure 5 Data Analysis: UCVA pre vs UCVA post.

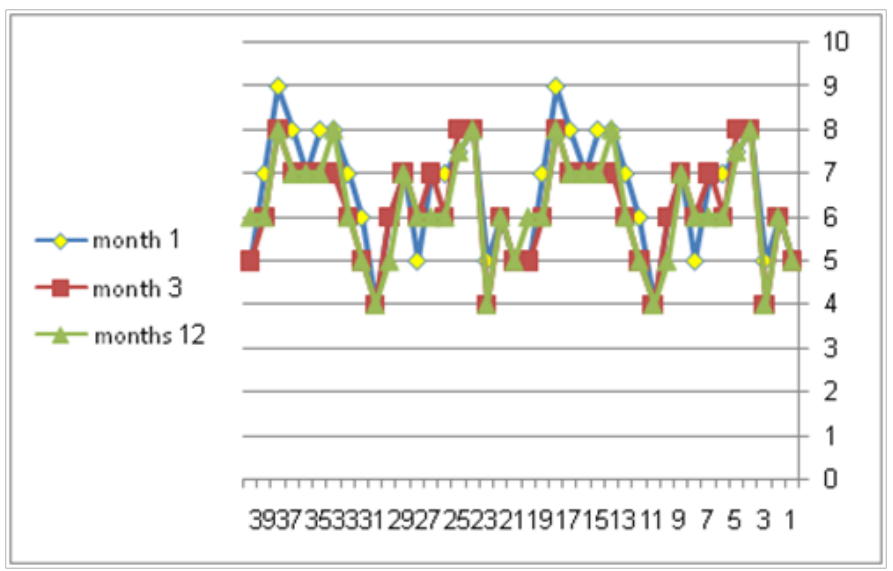

Figure 6 Effective procedure for pt with myopic astigmatism post PKP.

\section{Take home message}

I. Talk to the patient.

II. Determine his needs, expectation

III. Use the best and safest technology available to serve the best outcomes.

\section{Acknowledgments}

None.

\section{Conflicts of interest}

The authors declare that there is no conflict of interest.

\section{Funding}

None. 